\title{
Claudel et Whitman
}

\author{
Nina Hellerstein \\ University of Georgia
}

Comme la plupart des grands créateurs, Claudel n'aime pas avouer la véritable étendue de ses dettes à ses aînés. Ainsi, ses références à l'influence de Walt Whitman sont généralement enthousiastes, mais peu révélatrices de ce qu'a pu être le rôle du poète américain dans la formation de l'esthétique poétique claudélienne. Claudel ne semble pas le considérer comme un de ses grands précurseurs, à l'égal des illustres figures européennes qu'il cite souvent, Eschyle, Dante, Shakespeare, Rimbaud ou Mallarmé. Dans une lettre de 1916, il évoque l'influence que Whitman a exercée sur lui dans des termes positifs, mais généraux :

Je connais et j'admire de longue date Walt Whitman. C'est, je crois, mon ami Marcel Schwob qui le premier m'a fait lire les Leaves of Grass. Je ne me rends pas compte cependant qu'il a exercé une grande influence ni sur mes idées, ni sur ma technique, toute instinctive, et qui à ce moment était déjà fixée puisque j'avais écrit Tête d'Or et un autre drame depuis brûlé. (qtd in Erkkila 119)

Les travaux de Pierre Brunel ont reconstitué les circonstances de la rencontre de Claudel avec l'œuvre de Whitman, et nous savons qu'il possédait un exemplaire des Leaves of Grass acheté pendant son premier séjour américain et daté de 1894. L'on peut retrouver des traces thématiques importantes du poète américain dans le premier Echange, mettant en relief surtout la célébration whitmanienne de la nature et de l'aventure (Brunel L'Échange 44). Pourtant, comme le remarque Brunel, au-delà des rapprochements thématiques précis, il faut s'avancer avec prudence lorsqu'il s'agit d'affirmer une influence plus importante de Whitman sur la forme et les idées de Claudel (Brunel «L'Orchestre " 49-50).

L'on sait que les innovations whitmaniennes dans le domaine de la versification ont joué un rôle d'exemple dans les recherches des poètes 
symbolistes aboutissant à l'invention du vers libre, comme l'attestent des témoignages contemporains, dont celui d'Édouard Dujardin (606). Parmi ces témoignages, certains, comme Gide, identifient la poésie de Claudel comme particulièrement influencée par Whitman (Mansell-Jones 136). Pour pousser plus loin l'enquête sur les points possibles de rencontre entre les deux poètes, l'on peut examiner d'abord leurs idées sur le problème de la forme poétique et la nécessité de libérer la forme du vers. Déjà au milieu du $19 \mathrm{e}$ siècle, Whitman avait exprimé l'opinion que les formes traditionnelles de versification n'étaient plus un moyen valable d'expression pour la poésie moderne.

In my opinion [...] the time has arrived to essentially break down the barriers of form between prose and poetry. I say the latter is henceforth to win and maintain its character regardless of rhyme, and the measurementrules of iambic, spondee, dactyl, etc., and that [...] the truest and greatest Poetry (while subtly and necessarily always rhythmic, and distinguishable easily enough) can never again, in the English language, be express'd in arbitrary and rhyming metre. (qtd in Mansell-Jones «Symbolists », 61)

Lorsque Whitman rejette la rime et la régularité versifiée comme arbitraires et artificielles, lorsqu'il insiste sur le fait que la véritable poésie ne passe plus par les formes traditionnelles, désormais périmées, et qu'il est maintenant nécessaire de rapprocher les formes de la poésie et de la prose, il est très proche des conceptions de Claudel, comme celui-ci les a formulées explicitement dans ses « Réflexions et Propositions sur le Vers Français » de 1925.

Et cependant la poésie et la prose sont arrivées aujourd'hui à un point de développement où elles gagneraient à marier leurs ressources [...] Tout ce qu'il y a en français d'invention, de force, de passion, d'éloquence, de rêve, de verve, de couleur, de musique spontanée, de sentiment des grands ensembles, tout ce qui répond le mieux en un mot à l'idée que depuis Homère on se fait généralement de la poésie, chez nous ne se trouve pas dans la poésie, mais dans la prose. (Prose 43) 


\section{Claudel et Whitman}

C'est une formulation tardive des principes qui ont poussé Claudel, à partir de 1895, à se lancer dans une période d'expérimentation poétique dont les poèmes en prose de Connaissance de l'Est, les alexandrins des Vers d'exil et le verset lyrique des Cinq Grandes Odes sont le fruit.

L'illustration la plus directe du rapport étroit entre l'art des deux poètes est le parallèle entre la forme du vers whitmanien dans Leaves of Grass et le verset des Cinq Grandes Odes. Ils ont été influencés tous les deux par le verset biblique, avec son ton oraculaire et son emploi fréquent de répétitions et de parallélismes. La structure du verset whitmanien et celle du verset claudélien sont très proches, dans leur remplacement de la régularité métrique traditionnelle par des pieds ou groupements rythmiques plus souples, basés sur une organisation interne subtile des accents d'intensité.

On retrouve en somme là "l'iambe fondamental " de Claudel. Ce qui compte pour Whitman, comme pour Claudel, c'est le retour à intervalles à peu près réguliers de syllabes accentuées séparées les unes des autres par un nombre variable de syllabes non-accentuées (de une à quatre). (Asselineau 512-13)

Certains traits de cette forme commune créent la sensation d'une ressemblance remarquable entre les voix qui émanent des deux œuvres. L'un de ces traits les plus caractéristiques et les plus spectaculaires est l'emploi fréquent de l'apostrophe, combinée avec la répétition d'anaphores et d'exclamations qui commencent avec « $\mathrm{O}$ !»

$\mathrm{O}$ all and each well-loved by me! my intrepid nations! $\mathrm{O}$

I at any rate include you all with perfect love!

I cannot be discharged from you! not from one any sooner than another! (LG 21).

- O part! ô réservée! ô inspiratrice! ô partie réservée de moi-même! ô partie antérieure de moi-même!

$O$ idée de moi-même qui étais avant moi! (Euure poétique 273$)^{1}$

Ce procédé s'explique par l'importance de l'aspect oral, de la communication directe avec le lecteur, dans les deux ceuvres. Non 


\section{Nina Hellerstein}

seulement l'emploi des exclamations, mais aussi de nombreuses autres techniques poétiques renforcent cette impression. Par exemple, les deux poètes font un usage fréquent des formes interrogatives.

Will the whole come back then?

Can each see signs of the best by a look in the lookingglass? is there nothing greater or more? (199)

Est-ce que l'on bêche la mer? est-ce que vous la fumez comme un carré de pois?

Est-ce que vous lui choisissez sa rotation, de la luzerne ou du blé ou des choux ou des betteraves jaunes ou pourpres? (236)

L'impression de ressemblance entre ces deux passages vient de l'emploi parallèle du rythme et de l'organisation des vers : le rythme de la première question brève, qui lance la série interrogatoire, monte vers un sommet d'intensité, et l'effet est prolongé par les questions qui suivent, qui le répètent en l'agrandissant.

Cette importance de la communication orale et directe se retrouve dans un autre trait stylistique qui suggère une influence whitmanienne dans les Odes. Il s'agit de l'emploi des parenthèses :

As for me, (torn, stormy, amid these vehement days,)

I have the idea of all, and am all and believe in all,

I believe materialism is true and spiritualism is true, I reject no part.

(Have I forgotten any part? any thing in the past?

Come to me whoever and whatever, till I give you recognition.) (222)

Alors ne permettez point à celle-ci qu'elle vienne me tenter comme un jeune homme,

Non point avec un chant et avec la beauté de son visage,

(Où la suivrai-je, qui au bout de quatre pas n'est plus là?) (271) 
Dans les deux exemples, la parenthèse crée une sorte de distance ou de détachement momentané qui a l'effet de mettre en relief l'idée ainsi isolée, et surtout d'intensifier le sentiment de contact intime entre poète et lecteur. ${ }^{2}$

Cet effort d'évoquer une sensation de contact direct est basée sur l'idée, commune aux deux poètes, que les sources de la poésie sont physiques et organiques aussi bien que spirituelles. Whitman lui-même décrit la structure de sa poésie comme « a perpetual series of what might be called ejaculations " (qtd in Mansell-Jones «Vers Libre »135) : cette métaphore insiste à la fois sur la qualité dynamique de ses exclamations, leur rythme rapide, et sur la base corporelle de sa création, avec un parallèle entre l'activité érotique et la création poétique. En fait, la célébration du corps est un thème constant à travers Leaves of Grass. L'on retrouve cette même insistance sur la base organique de la poésie à travers l'œuvre claudélien, notamment dans Art poétique, et elle s'exprime dans les Odes par une allusion à la création poétique comme un acte organique où le corps et l'esprit collaborent, un effort intense de l'être entier qu'il compare à la procréation dans la Quatrième Ode.

Ce n'est point avec le tour et le ciseau que l'on fait un homme vivant, mais avec une femme, ce n'est pas avec l'encre et la plume que l'on fait une parole vivante! (268)

Ces métaphores insistent sur la qualité d'immédiateté physique qui caractérise la poésie de Whitman et de Claudel. Elle est fondée sur la croyance que le langage lui-même est organique et créateur par nature, et que le poète doit exploiter ces qualités au lieu de chercher à les supprimer en emprisonnant le langage dans des règles arbitraires et des lois mécaniques de composition. Les deux poètes condamnent les règles rigides des grammairiens et approuvent l'emploi de formes familières, grammaticalement douteuses ou même incorrectes. Whitman insiste particulièrement sur les puissances génératives de l'argot, qu'il considère comme une ressource précieuse et essentielle : « the lawless germinal element, below all words and sentences, and behind all poetry » (qtd in Warren 110). Claudel critique la mentalité étroite et restrictive des grammairiens tyranniques, incapables de reconnaître l'instinct musical 


\section{Nina Hellerstein}

naturel et inné dans tout langage parlé : « La voix a ses lois, l'âme a ses exigences, qui ne sont pas celles de la logique et de l'écriture »(Prose 41).

Cette croyance à la liberté linguistique et poétique est la base de la syntaxe très personnelle de Leaves of Grass aussi bien que de l'œuvre claudélien, et contribue à l'impression de similarité dans la voix des deux poètes. Par exemple, tous les deux font un usage fréquent et très particulier du participe présent. Malgré le fait que « la langue française n'aime pas beaucoup [le participe présent], presque banni de la diction poétique ", comme le montre Jean Catel (119), il y a de nombreux passages dans les Cinq Grandes Odes qui sont construits sur des participes présents.

Mais maintenant, quittant le niveau de la mer liquide,

O rimeur Florentin! nous ne te suivrons point, pas après pas, dans ton investigation,

Descendant, montant jusqu'au ciel, descendant jusque dans l'Enfer,

Comme celui qui assurant un pied sur le sol logique avance l'autre en une ferme enjambée. (224)

Dans le participe présent, la préférence claudélienne pour le verbe et le dynamisme actif peut se combiner avec un sens de simultanéité, évoquant un système de rapports dynamiques et synthétiques entre les êtres, suivant ses idées dans Art poétique. Il invente même une syntaxe plus synthétique pour mieux exprimer ce dynamisme d'origine divine, évoqué dans l'adjectif participial :

Afin que pas ce rayon de votre lumière vie-créante qui m'était destiné n'échappe. (239)

Bien que le participe présent soit plus normal dans la poésie de langue anglaise, Whitman en fait un usage exceptionnel, pour des raisons proches de celles de Claudel : un sens d'activité verbale où l'identité personnelle du poète s'efface pour rehausser le dynamisme inhérent aux choses (Catel 119-21). C'est particulièrement évident dans ses célèbres catalogues, des énumérations d'êtres, d'actions ou de noms.

The blows of mallets and hammers, the attitudes of the men, their curv'd limbs, 


\section{Claudel et Whitman}

Bending, standing, astride the beams, driving in pins, holding on by posts and braces,

The hook'd arm over the plate, the other arm wielding the axe,

The floor-men forcing the planks close to be nail'd,

Their postures bringing their weapons downward on the bearers,

The echoes resounding through the vacant building. (169)

La technique du catalogue est un autre trait du style whitmanien qui se retrouve dans les Odes : Claudel y utilise à plusieurs reprises des techniques d'accumulation et d'énumération qui rappellent beaucoup cette structure. On les trouve associées à une sorte d'entassement creux et matérialiste dans l'accumulation des noms "païens ", vides et idolâtriques, stigmatisés dans « Magnificat »:

Soyez béni, mon Dieu, qui m'avez délivré des idoles,

Et qui faites que je n'adore que Vous seul, et non point Isis ou Osiris,

Ou la Justice, ou le Progrès, ou la Vérité, ou la Divinité, ou l'Humanité, ou les Lois de la Nature, ou l'Art, ou la Beauté, [...]

Je n'honorerai point les fantômes et les poupées, ni Diane, ni le Devoir, ni la Liberté et le bouf Apis. (251-2)

Ces catalogues négatifs s'opposent directement à une série d'énumérations positives, qui associent l'eau et les fleuves avec le thème de l'unité vitale des forces terrestres :

C'est moi, je tire, j'appelle sur toutes mes racines, le Gange, le Mississipi,

L'épaisse touffe de l'Orénoque, le long fil du Rhin, le Nil avec sa double vessie, [...]

(O les détroits de Malaisie où roule un arbre noir couvert d'oiseaux! détroit de Banda! mer de Sulu où naviguaient 


\section{Nina Hellerstein}

les vieilles hourques hollandaises, grosses et dures comme une noix vernie! ô les premières gouttes de l'averse qui roulent dans la poussière de la pluie de l'Équateur pareille à du rhum tiède!) $(237,286)$

L'on retrouve l'influence whitmanienne dans cette importance des noms des mers et des fleuves, qui représentent l'unité et la diversité des endroits du monde entier; dans l'œuvre de Whitman, c'est associé en particulier avec les noms indiens, qui évoquent une géographie américaine primordiale.

Okonee, Koosa, Ottawa, Monongahela, Sauk, Natchez, Chattahoochee, Kaqueta, Oronoco, Wabash, Miami, Saginaw, Chippewa, Oshkosh, WallaWalla. (22)

Déjà dans Connaissance de l'Est, Claudel se souvenait de ce trait dans l'exotique « rivière Haha » de « La Dérivation », et dans la Quatrième Ode, où il évoque « le canot démontable qui remonte l'Aruwhimi » (59, 267). Ce thème évoque les puissances créatrices de la nature, comme on le voit par son association avec les fluides organiques dans Connaissance de l'Est (62). Et le thème de l'unité du monde humain et naturel rappelle que les catalogues de Whitman sont associés avec son sentiment de l'unité panthéiste et démocratique du monde (Allen 384-5).

Claudel a pu trouver dans cette pratique de Whitman un écho de ses idées sur la puissance créatrice du langage lui-même : on a vu dans les catalogues whitmaniens une interrogation des pouvoirs du langage et un effort de créer en nommant, dans une sorte de "magie verbale " ("word magic ") (Nathanson 145-6). La question des puissances du langage renvoie au statut du poète lui-même, et ici aussi, il y a des parallèles intéressants entre la vision des deux vis-à-vis de leur propre rôle. En fait, derrière leur insistance sur la liberté linguistique et poétique, derrière leur préoccupation avec la voix poétique et ses pouvoirs, l'on retrouve une croyance commune à la mission quasi-divine du poète. Les deux exaltent son rôle et construisent une persona qui renvoie à l'image romantique du mage prophétique et inspiré. Whitman se présente comme le Barde, héritier des traditions anciennes, tandis que le poète des Cinq Grandes Odes se compare à Homère, Virgile et Dante. En fait, les passages les plus « whitmaniens » des Cinq Grandes Odes sont aussi les plus épiques en ton 


\section{Claudel et Whitman}

et en thème, lorsque le poète célèbre les œuvres de l'humanité et exalte son propre rôle de participant à ces accomplissements. Et surtout, Whitman offre à Claudel le modèle d'une voix essentiellement positive, optimiste et enthousiaste qui est en même temps totalement moderne et qui prend la réalité contemporaine comme son objet.

See, steamers steaming through my poems, [...]

See, the many-cylinder'd steam printing-press - see, the electric telegraph stretching across the continent, $[\ldots]$

See, the strong and quick locomotive as it departs, panting, blowing the steam-whistle,

See, ploughmen ploughing farms - see, miners digging mines - see, the numberless factories [...] (23)

La première Strophe de la Quatrième Ode exprime une célébration très semblable de l'industrie, des transports et de la technologie modernes (Erkkila 133) :

Ce que les gens ont fait autour de moi avec le canon qui ouvre les vieux Empires,

Avec le canot démontable qui remonte l'Aruwhimi, avec l'expédition polaire qui prend des observations magnétiques,

Avec les batteries de hauts fourneaux qui digèrent le minerai, avec les frénétiques villes haletantes et tricotantes [...]

Je le ferai avec un poëme qui ne sera plus l'aventure d'Ulysse parmi les Lestrygons et les Cyclopes, mais la connaissance de la Terre... (267)

Il est intéressant de noter à propos de ce thème qu'en 1892, Claudel était moins enthousiaste sur la valeur de la technologie dans l'œuvre de Whitman.

Comme moi aussi j'aime les vers de Whitman, [...] il y a une chose que je ne lui pardonne pas; il a chanté une exposition et il croit au progrès par la science et par les babioles semblables. (Martin-Schmets 153). 


\section{Nina Hellerstein}

$\mathrm{Si}$, dix ans plus tard dans les Odes, l'attitude du poète est plus favorable aux entreprises humaines de conquête matérielle, c'est peut-être qu'il a commencé à regarder la réalité pratique de la vie comme un thème plus intéressant. Dans une lettre datant de la même époque, il parle de sa joie de « toucher à toutes ces choses bien grosses et bien réelles, tramways, égouts, électricité, et l'impitoyable comptabilité » (O.C. 226). En tout cas, plus tard dans sa vie, après des années d'expérience de la vie pratique, il exprime son admiration pour le poète américain et pour son sens de la fraternité internationale, « ces frères sur toute la planète qu'on mettait à notre disposition ", qu'il associe à la foi dans le changement, le progrès technologique et "l'œuvre positive du XIXe siècle » dans sa «Conversation» sur Richard Wagner (Prose, 865-6, qtd in Erkkila 137).

Ce dernier passage évoque un thème qui forme peut-être le lien le plus profond entre la pensée des deux poètes : celui de l'unité du monde et la dimension globale, cosmique de leur vision poétique. L'œuvre de Claudel repose sur l'idée que le poète embrasse la totalité de l'univers dans son système d'analogies et de rapports poétiques. C'est aussi un concept d'importance majeure dans la poésie de Whitman.

A vast similitude interlocks all,

All spheres, grown, ungrown, small, large, suns, moons, planets, [...]

All identities that have existed or may exist on this globe, or any globe, [...]

This vast similitude spans them, and always has spann'd [...] (240)

Ce point final d'intersection nous amène à conclure que la poésie de Whitman représente une inspiration positive, un modèle optimiste pour Claudel, dans la plupart de ses thèmes et de ses principes esthétiques. Pourtant, il y a un aspect plus sombre et ambigu associé avec cette influence. Il est significatif, par exemple, que Louis Laine, le plus whitmanien des personnages de Claudel, finit mal. En plus, les héros les plus épiques de Claudel n'atteignent pas la gloire qu'ils cherchent à travers l'aventure et la conquête. Les deux poètes sont fascinés par la figure de Christophe Colomb, mais il est significatif que les deux choisissent d'insister sur la fin tragique et solitaire de sa vie. La célébration whitmanienne du moi et de la voix poétique est en fait une construction 


\section{Claudel et Whitman}

extrêmement complexe qui comprend de nombreux avertissements au lecteur sur l'ambiguité et le caractère fuyant de la persona poétique de Leaves of Grass (voir l'ouvrage de Nathanson). De la même façon, la persona poétique de Connaissance de l'Est et des Cinq Grandes Odes est une figure complexe qui combine la célébration de la fonction poétique et une interrogation douloureuse sur le rôle du moi personnel dans cette fonction. L'on retrouve peut-être dans cet aspect énigmatique de l'œuvre claudélien un souvenir du précurseur génial qui a légué aux lettres modernes un héritage si riche.

\section{Notes}

1 L'on notera que le même emploi de l'exclamation précédée par « $\mathrm{O}$ ! » se trouve chez Coventry Patmore, quoique plus rarement. Voir Maurocordato Anglo-American 78. Voir aussi Antoine, 40-2 pour ce procédé.

2 On trouve dans les ouvrages de Catel (126-8) et de Allen (417-20) des discussions de ce trait dans l'œuvre de Whitman; pour l'emploi claudélien, voir Maurocordato L'Ode (64). Il faut noter que le procédé est beaucoup plus fréquent chez Whitman.

\section{Curvres citées}

Allen, Gay Wilson. Walt Whitman Handbook. New York : Hendricks House, 1962.

Antoine, Gérald. Les Cinq Grandes Odes de Claudel ou la poésie de la répétition. Paris : Lettres Modernes, 1959.

Asselineau, Roger. L'Évolution de Walt Whitman. Paris : Didier, 1954.

Bernard, Suzanne. Le Poème en prose de Baudelaire jusqu'à nos jours. Paris : Nizet, 1959.

Brunel, Pierre. «L'Échange » de Paul Claudel. Besançon : Annales Littéraires de l'Université de Besançon, 1974.

- "L'Image de l'Orchestre et la Tentation Symphonique chez Whitman et Claudel », La Revue des Lettres Modernes 134-36 (1966), 49-63.

Catel, Jean. Rythme et langage dans la première édition des "Leaves of Grass », Paris : Rieder, 1952. 


\section{Nina Hellerstein}

Claudel, Paul. Euvres en prose. Ed. J. Petit et C. Galpérine. Paris : Gallimard, «La Pléiade ", 1965.

- CEurre poétique. Ed. S. Fumet et J. Petit. Paris : Gallimard, «La Pléiade », 1967.

- Euvres complètes, Vol. XV. Ed. R. Mallet. Paris : Gallimard, 1959.

Dujardin, Edouard. "Les Premiers Poètes du Vers Libre ", Mercure de France 15 mars 1921, p. 577-621.

Erkkila, Betsy. Walt Whitman among the French. Princeton : Princeton UP, 1980. Mansell-Jones, P. «Whitman and the Symbolists », French Studies II, 1 (1948), 54-67.

— "Whitman and the Origins of the 'Vers Libre'», French Studies II, 2 (1948), 129-139.

Martin-Schmets, Victor. «Un Article de Byvanck sur Claudel », La Revue des Lettres Modernes 616-20 (1981), 146-58.

Maurocordato, Alexandre. Anglo-American Influences in Paul Claudel. Genève : Droz, 1964.

- L'Ode de Paul Claudel, I. Paris : Lettres Modernes, 1974.

Nathanson, Tenney. Whitman's Presence. New York : NYUP, 1992.

Warren, James Perrin. Walt Whitman's Language Experiment. University Park, Pa. : Penn State UP, 1990.

Whitman, Walt. Leaves of Grass. New York : Heritage Club, 1937. 